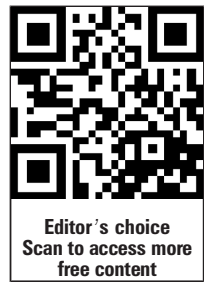

free content

ORIGINAL RESEARCH

\title{
Angioarchitectural features associated with hemorrhagic presentation in pediatric cerebral arteriovenous malformations
}

\author{
Michael J Ellis, ${ }^{1}$ Derek Armstrong, ${ }^{2}$ Shobhan Vachhrajani, ${ }^{1}$ \\ Abhaya V Kulkarni, ${ }^{1}$ Peter B Dirks, ${ }^{1,2}$ James M Drake, ${ }^{1}$ Edward R Smith, ${ }^{3}$ \\ R Michael Scott, ${ }^{3}$ Darren B Orbach ${ }^{4}$
}

${ }^{1}$ Department of Neurosurgery, Hospital for Sick Children, Toronto, Ontario, Canada ${ }^{2}$ Department of Neuroradiology, Hospital for Sick Children, Toronto, Ontario, Canada ${ }^{3}$ Department of Neurosurgery, Children's Hospital Boston, Boston, Massachusetts, USA ${ }^{4}$ Department of Neurointerventional Radiology. Children's Hospital Boston, Boston, Massachusetts, USA

\section{Correspondence to}

Dr D B Orbach, Department of Neurointerventional Radiology, Children's Hospital Boston, 300 Longwood Avenue, Boston, MA 02115, USA; darren.orbach@ childrens.harvard.edu

Received 16 November 2011 Revised 16 February 2012 Accepted 23 February 2012

Published Online First 13 March 2012

\begin{abstract}
Background and objective To date, there have been few published studies examining the relationship between arteriovenous malformation (AVM) angioarchitecture and hemorrhagic presentation among children with cerebral AVMs. This study examines this relationship in this unique population, in whom symptomatic presentation of cerebral AVM is the norm rather than the exception.

Methods A cohort of children with AVMs from 2000 to 2011 were included. Predictors studied included patient age, gender and angioarchitectural features, including AVM location, nidus size and morphology, venous drainage, presence of venous outflow lesions and associated aneurysms. Predictors of hemorrhagic presentation were assessed using multivariate logistic regression.
\end{abstract}

Results 135 children (70 males, mean age 10.1 years) were included. 86/135 (63.7\%) children presented with hemorrhage, 18 (13.3\%) with seizures, 17 (12.6\%) with headaches or neurological deficits and $14(10.4 \%)$ were asymptomatic. AVM location, morphology and the presence of associated aneurysm, venous ectasia, draining vein stenosis and single draining vein were not significantly associated factors. After multivariate analysis, AVM size (OR $0.57,95 \% \mathrm{Cl} 0.43$ to 0.77 ; $\mathrm{p}<0.01$ ), exclusive deep venous drainage (OR $4.94,95 \%$ $\mathrm{Cl} 1.30$ to 18.8; $\mathrm{p}=0.02$ ) and infratentorial location (OR 9.94, $95 \% \mathrm{Cl} 1.71$ to $51.76 ; \mathrm{p}=0.01$ ) were

independently associated with hemorrhagic presentation. Conclusion Smaller AVM size, exclusive deep venous drainage and infratentorial location are specific angioarchitectural factors independently associated with initial hemorrhagic presentation in children with AVMs.

\section{INTRODUCTION}

Management of cerebral arteriovenous malformations (AVMs) in children requires careful consideration of treatment related risks weighed against the natural history of the lesion, including the risk of intracranial hemorrhage. While the past few decades have brought remarkable improvements in neuroimaging and the therapeutic armamentarium with which we approach these lesions, few advances have been made in our understanding of the natural history and clinical behavior of pediatric AVMs.
Studies in adults have identified specific angioarchitectural features of AVMs that are associated with hemorrhagic presentation and future hemorrhage. Small size, deep location, deep venous drainage, single draining vein, intranidal aneurysms and associated venous abnormalities such as ectasia and stenosis have all been implicated as morphological risk factors of hemorrhage. ${ }^{1-11}$ Similar studies in children are lacking and treatment decision making is therefore often extrapolated from adult data.

This study aims to associate the presence of specific angioarchitectural features with hemorrhagic presentation among a cohort of children with AVMs managed at two pediatric neurosurgery referral centers.

\section{METHODS}

We retrospectively reviewed the demographic, clinical and radiological characteristics of all patients ( $\leq 19$ years of age) with an AVM who were referred to us between January 2000 and March 2011. Only patients who underwent cerebral angiography and cross sectional imaging prior to AVM directed treatment were included. All patients with spinal AVMs, vein of Galen aneurysmal malformations, dural arteriovenous fistulae or single hole pial arteriovenous fistulae were excluded from the study. Research ethics boards at both institutions approved the protocol for this study.

Patient age at presentation, gender, past medical history and clinical presentation were recorded Angioarchitectural features were analyzed using the methodology established by the Joint Writing Group of the Technology Assessment Committee, 2001. ${ }^{12}$ AVM characteristics, including location, diameter and venous drainage, were recorded and graded using the Spetzler-Martin grading scale. ${ }^{13}$ AVM size was classified as: small $(<3 \mathrm{~cm})$, medium $(3-6 \mathrm{~cm})$ and large $(>6 \mathrm{~cm})$ for the purpose of descriptive statistics but was analyzed as a continuous variable for the purpose of univariate and multivariate analysis. AVM location, based on nidus location, was classified in two different ways: deep (located in the basal ganglia, thalamus, brainstem or corpus callosum) versus superficial (all other locations) and supratentorial versus infratentorial. AVM nidus morphology was subjectively classified as compact (presence of little or no 
intervening brain within the AVM nidus), diffuse (presence of significant intervening brain within the AVM nidus) or fistulous (predominant AV shunting with little intervening nidus). Venous drainage was classified as superficial, deep or both, and lesions were evaluated for the presence of single or multiple draining veins. Venous pouches were defined as focal aneurysmal dilatations of the proximal draining vein. Venous ectasia was defined as a greater than twofold increase in a segment of the draining vein along its course from the nidus to its venous collector. Venous stenosis was defined in two ways: angiographic venous stenosis (defined as $>50 \%$ narrowing of the draining vein or venous system) and functional venous stenosis (defined as the persistence of contrast within the AVM nidus and draining vein at the time of visualization of the normal cortical or cerebellar venous drainage). Venous sinus abnormalities that contributed to venous outflow obstruction (ie, sinus thrombosis) were also characterized. Associated aneurysms were described based on their relationship to the AVM nidus (prenidal, intranidal or remote).

\section{Statistics}

Descriptive statistics were used to characterize the study population. The Student's t test was used to compare means between groups, and Pearson's $\chi^{2}$ and Fisher's exact tests were used for comparisons of categorical variables where appropriate. Univariate logistic regression analysis was used to identify potential independent predictors of hemorrhagic presentation. Variables previously examined in the adult literature were initially chosen for an exploratory univariate analysis of potential features associated with hemorrhagic presentation in children. The Harrell method ${ }^{14}$ was subsequently used to build a multivariate logistic regression model. Statistical significance was set at $\mathrm{p}<0.05$. All statistics were performed using SAS V.9.2 for Windows.

\section{RESULTS}

The study included 135 children (70 males and 65 females): 89 $(66 \%)$ from the Hospital for Sick Children, Toronto, and 46 (34\%) from the Children's Hospital Boston. Twenty-one children were excluded from the study based on incomplete neuroimaging or previous AVM directed therapy. Mean age at presentation was 10.1 years (range $0-19$ years). There was no significant difference in the mean age of those presenting with and without hemorrhage (10.3 vs 9.7 years; $p=0.42)$. Eighty-six of $135(63.7 \%)$ children presented with hemorrhage, 18 (13.3\%) with seizures, $17(12.6 \%)$ with headaches or neurological deficits and 14 (10.4\%) were asymptomatic. Seven (5.2\%) patients had a documented diagnosis of hereditary hemorrhagic telangiectasia but none presented with hemorrhage. The frequency of hemorrhage among those patients with specific angioarchitectural features is summarized in table 1. One patient demonstrated AVM venous drainage into a thrombosed venous sinus.

Factors not significantly associated with hemorrhagic presentation included patient age, gender, AVM location, morphology and the presence of venous ectasia or pouch, associated aneurysm, angiographic and functional venous stenosis, and single draining vein. Univariate analysis revealed four features to be significantly associated with initial hemorrhagic presentation: smaller AVM size, exclusive deep venous drainage, infratentorial location and venous obstruction (the composite presence of any of venous ectasia, functional venous stenosis, single draining vein and draining venous sinus thrombosis).
Table 1 Frequency of hemorrhage as a function of angioarchitectural features in pediatric arteriovenous malformations

\begin{tabular}{llrr}
\hline Feature & Subgroup & Total No & $\begin{array}{l}\text { No with } \\
\text { hemorrhage (\%) }\end{array}$ \\
\hline Location & Superficial & 117 & $72(61.5)$ \\
& Deep & 18 & $14(77.8)$ \\
& Supratentorial & 119 & $72(60.5)$ \\
Size $(\mathrm{cm})$ & Infratentorial & 16 & $14(87.5)$ \\
& $<3$ & 88 & $64(72.7)$ \\
Venous drainage & $3-6$ & 43 & $21(48.8)$ \\
& $>6$ & 4 & $1(25.0)$ \\
Associated aneurysm & Superficial only & 82 & $49(59.8)$ \\
& Deep only & 23 & $20(87.0)$ \\
Venous pouch & Both & 30 & $17(56.7)$ \\
Draining veins & Intranidal & 7 & $6(85.7)$ \\
& Prenidal & 4 & $3(75.0)$ \\
Venous ectasia & None & 124 & $76(61.8)$ \\
& Present & 15 & $8(53.3)$ \\
Angiographic venous stenosis & None & 120 & $78(65.0)$ \\
& Single & 66 & $47(71.2)$ \\
Functional venous stenosis & Multiple & 69 & $39(56.5)$ \\
& Present & 5 & $3(60.0)$ \\
Nidus morphology & None & 130 & $83(63.9)$ \\
& None & 24 & $17(70.8)$ \\
& Present & 111 & $69(62.2)$ \\
& None & 18 & $15(83.3)$ \\
& Compact & 91 & $71(60.7)$ \\
& Diffuse & 33 & $39(53.8)$ \\
& Fistulous & 10 & $6(60.0)$ \\
\hline
\end{tabular}

Only smaller AVM size, exclusive deep venous drainage and infratentorial location were independently associated with hemorrhagic presentation in multivariate analysis (table 2).

\section{DISCUSSION}

Studies aimed at identifying angioarchitectural features associated with hemorrhagic presentation and with risk of future hemorrhage in patients with AVMs have been largely limited to single institution studies involving predominantly adult patients. As a result, little is known about such relationships in children with AVMs. To date, only one published abstract has addressed this issue in an exclusive pediatric cohort. Kellner et $a l^{15}$ studied the morphology of AVMs in 77 children referred to Columbia University Medical Center (CUMC), $62 \%$ of whom presented with hemorrhage. Small AVM size, eloquent location and deep venous drainage were independently associated with AVM hemorrhage.

To fully characterize this relationship, we evaluated the clinical and angioarchitectural features in a combined exclusively pediatric cohort with AVMs referred to two high volume pediatric neurosurgery centers over the past 11 years. Our multivariate analysis yielded smaller AVM size, exclusive deep venous drainage and infratentorial location as being independently associated with initial hemorrhagic presentation.

Table 2 Multivariate analysis of angioarchitectural features associated with hemorrhagic presentation

\begin{tabular}{llc}
\hline Feature & $\mathbf{O R}(\mathbf{9 5} \% \mathbf{C l})$ & $\mathbf{p}$ Value \\
\hline Size & $0.57(0.43$ to 0.77$)$ & $<0.01$ \\
Exclusive deep drainage & $4.94(1.30$ to 18.8$)$ & 0.02 \\
Infratentorial location & $9.94(1.71$ to 51.76$)$ & 0.01 \\
\hline
\end{tabular}




\section{Smaller AVM size}

Several adult studies have identified small AVM size $(\leq 3 \mathrm{~cm})$ as a risk factor for hemorrhage. Guidetti and Delitala, ${ }^{16}$ Itoyama et $a l^{17}{ }^{17}$ Graf et $a l^{18}$ and Crawford et al ${ }^{19}$ all observed a higher rate of hemorrhagic presentation among smaller versus larger AVMs. Although the pathophysiological mechanisms underlying this association remain unclear, some authors have postulated a relationship between AVM size and feeding artery pressures. Spetzler et $a l^{20}$ found a higher rate of hemorrhagic presentation among smaller AVMs and noted that smaller AVMs were associated with higher feeding artery pressures at the time of surgical management as well as larger hematoma sizes. Despite the high rates of hemorrhagic presentation observed among small AVMs, several prospective studies have failed to find an association between AVM size and future hemorrhage. 239

In the present study, we found a higher rate of hemorrhagic presentation among small AVMs (72.7\%) compared with medium sized (48.8\%) and large AVMs (25\%), and smaller AVM size was independently associated on multivariate analysis. These results corroborate those of the CUMC study and suggest that smaller AVM size is a potentially important risk factor for hemorrhagic presentation in children with AVMs.

\section{Deep venous drainage}

The presence of deep venous drainage is among the most consistently reported features associated with hemorrhage in adult AVMs. Marks et al ${ }^{6}$ found hemorrhagic presentation more frequently in patients with central venous drainage (94\%) over cortical (37\%) or mixed drainage (50\%). Miyasaka et al ${ }^{21}$ found a similar relationship, with $94 \%$ of AVMs with exclusive deep drainage presenting with hemorrhage. Because deep seated AVMs are more likely to have a component of deep venous drainage, previous studies have pointed out that the relationship between deep venous drainage and hemorrhage may be influenced by AVM location. Although one study identified deep location and not deep venous drainage as a significant factor at presentation, ${ }^{10}$ others have identified both deep drainage ${ }^{422}$ and exclusive deep drainage $e^{23}$ as independent predictors of hemorrhagic presentation. Prospective studies have also identified deep venous drainage $e^{7}$ and exclusive deep venous drainage ${ }^{9}$ as important risk factors for future AVM hemorrhage.

In this study, hemorrhagic presentation was observed in $87 \%$ of children with exclusive deep venous drainage, $56.7 \%$ with superficial and deep venous drainage, and $59.8 \%$ with superficial drainage alone. Moreover, exclusive deep venous drainage was independently associated with hemorrhagic presentation. We did not observe a relationship between deep location and hemorrhagic presentation in this study. Therefore, these findings, and those of the CUMC study, suggest that deep venous drainage is an important independent risk factor of hemorrhage in pediatric AVMs.

\section{Infratentorial location}

The role of location in AVM hemorrhage is unclear. Although comprising only $7-15 \%$ of intracranial AVMs, hemorrhage rates of infratentorial AVMs among adult surgical series range from $72 \%$ to $92 \%,{ }^{24}$ and studies have identified infratentorial location as independently associated with both initial ${ }^{5}$ and future ${ }^{3}$ AVM hemorrhage. This high hemorrhage rate has been explained by several factors, including the high frequency of perforator supply and exclusive deep venous drainage, ${ }^{3}$ as well as 'kinking' of the venous system at the level of the tentorium. ${ }^{11}$ Because infratentorial AVMs are less likely to present with seizures, some authors have suggested that this may contribute to the higher rates of hemorrhage observed among reported series.

Our study included 16 children with infratentorial AVMs, 14 $(87.5 \%)$ of whom presented with hemorrhage. Infratentorial location was found to be independently associated with hemorrhagic presentation. The true impact of this finding is difficult to determine, however, as this is the only study to implicate infratentorial location as an independent risk factor in children and has a sample size too small to make definitive conclusions.

\section{Associated aneurysms and venous pouches}

The incidence of arterial aneurysms associated with adult AVMs varies in the literature from $10 \%$ to $58 \%$ and is complicated by inconsistent definitions and variations in imaging techniques. ${ }^{1} 2825$ Multiple adult studies have established associated aneurysms as a risk factor for both initial hemorrhagic presentation and future hemorrhage. ${ }^{12} 6825$ Our study included only $11(8 \%)$ children with associated aneurysms (seven patients with intranidal, four patients with proximal flow related aneurysms), nine (81.8\%) of whom presented with hemorrhage. Despite this high rate of hemorrhage, this feature was not significantly associated even on univariate analysis, likely due to the small number of children with associated aneurysms. Importantly, arterial aneurysms can be difficult to discern from venous pouches which, although less studied, have also been implicated as a predisposing factor to AVM hemorrhage. In this study, 8/15 (53.3\%) children with associated venous pouches presented with hemorrhage although this feature was not independently predictive of hemorrhage.

\section{Venous drainage anatomy}

Several AVM features that either promote or reflect the development of venous outflow restriction and subsequent disruption of the transmural pressure gradient across AVMs have been studied. Specifically, studies among adults have noted higher rates of hemorrhage among AVMs with deep venous drainage obstruction, draining vein stenosis, a small number of draining veins and venous ectasias. ${ }^{321} 26-28$ On univariate analysis, the presence of venous obstruction (the presence of a single draining vein, venous ectasia or functional venous stenosis) was associated with a higher likelihood of hemorrhagic presentation. However, on multivariate analysis, no individual feature of the venous system (other than exclusive deep venous drainage) was found to be significant.

\section{AVM morphology}

AVMs display wide variability in their overall angiographic morphology and flow characteristics, and represent a continuum from high flow fistulous lesions to linear cylindrical lesions extending from the cortex towards the ventricle, ${ }^{29}$ to compact or glomerular-type lesions, to diffuse or proliferative lesions. In this study, we did not observe an association between nidus morphology and hemorrhagic presentation in children with AVMs.

\section{Study limitations and clinical implications}

Despite providing important insight into the natural history of pediatric AVMs, this study has several limitations. The most important of these is selection bias, which is common to all of the discussed studies and is impacted by both the study population and the referral center. Among adult neurosurgical centers, major differences in the demographic, clinical and morphological features of AVM patient populations have been observed. ${ }^{30}$ 
These differences may, in part, explain the wide variability in which AVM features are identified as risk factors of hemorrhage among reported studies. Although differences in the institutional populations were not formally assessed, certain referral biases were evident in this study. For instance, the greater proportion of hemorrhagic AVMs among the Hospital for Sick Children cohort (73\% vs $45 \%$ ) and higher rates of non-hemorrhagic AVMs among the Children's Hospital Boston cohort $(54 \%$ vs $27 \%$ ) may reflect differences in referral patterns between pediatric institutions.

A second important limitation of this study that is also common to the other discussed studies is sample size. While the present study analyzed angioarchitectural features in an 11 year combined cohort from two high volume pediatric neurosurgery centers, the relatively small sample size may be underpowered to assess the true impact of certain angioarchitectural features on AVM hemorrhage and may also not be able to fully control for the interaction between specific variables and the risk of false positive results in the setting of multiple comparisons.

A third important limitation to this study concerns the use of superselective angiography. Despite tremendous advances in conventional and three-dimensional rotational angiography, these techniques are still limited in their ability to characterize intranidal abnormalities such as arterial aneurysms and venous pouches. Because we did not exclude the small proportion of children that underwent superselective angiography for pretreatment planning of endovascular embolization, we may have overestimated the frequency of these features in this patient population. By the same token, we may have also failed to detect important intranidal pathology in those children that were not considered for endovascular therapy.

The final limitation concerns the clinical implications of this study. The true value of AVM natural history studies resides in their ability to discriminate between AVMs that carry a high risk of hemorrhage versus those with a lower risk of hemorrhage on the basis of AVM angioarchitecture. Future risk of hemorrhage can then be weighed against the risk of treatment to help guide therapeutic decision making. The extent to which the presence of certain morphological risk factors present at the time of hemorrhagic presentation can be extrapolated as predictors of future hemorrhage in AVMs is controversial. Although smaller AVM size, exclusive deep drainage and infratentorial location were found to be independently associated with hemorrhage in pediatric AVMs, this analysis represents only those factors significant at presentation and not necessarily those that predict future hemorrhage. While further prospective studies, as well as the ongoing ARUBA trial (A Randomized Trial of Unruptured Brain Arteriovenous Malformations, NCT00389181) will help clarify those features that are predictive of future hemorrhage in adults with AVMs, similar studies are unlikely to be completed among children where very few are treated conservatively. In our opinion, ruptured AVMs in children carry a significant risk of recurrent hemorrhage and associated morbidity and mortality and, as such, should be treated aggressively with the goal of complete AVM obliteration with minimal neurological morbidity. Although the management of unruptured AVMs remains controversial, we believe the presence of high risk angioarchitectural features should be strongly considered when weighing treatment related risks against the natural history of AVMs in children.

Despite these limitations, the present study provides important insight into the angioarchitectural features that are associated with hemorrhagic presentation in a large combined cohort of pediatric AVMs. If the morphological risks of future AVM hemorrhage are to be determined in the future, they will undoubtedly require the collaborative efforts of multiple pediatric neurosurgical centers.

\section{CONCLUSIONS}

In this combined cohort of 135 children with AVMs, smaller AVM size, exclusive deep venous drainage and infratentorial location were independently associated with initial hemorrhagic presentation on multivariate analysis. Future prospective collaborative studies are needed to confirm these findings and identify factors predictive of future hemorrhage in children with AVMs.

Contributors All authors contributed substantially to the text, by way of writing a draft, performing and critiquing the review of the relevant imaging, or constructing and critiquing the statistical analysis, and every author participated actively in editing and reworking the manuscript.

Funding Partial support for some of the Canadian investigators was provided by the Ontario Stroke System Research Program.

\section{Competing interests None.}

Ethics approval Ethics approval was provided by the institutional review board of the Hospital for Sick Children Toronto and the Children's Hospital Boston.

Provenance and peer review Not commissioned; externally peer reviewed.

\section{REFERENCES}

1. Brown RD Jr, Wiebers DO, Forbes GS. Unruptured intracranial aneurysms and arteriovenous malformations: frequency of intracranial hemorrhage and relationship of lesions. J Neurosurg 1990;73:859-63.

2. da Costa L, Wallace MC, Ter Brugge KG, et al. The natural history and predictive features of hemorrhage from brain arteriovenous malformations. Stroke 2009:40:100-5.

3. Hernesniemi JA, Dashti R, Juvela $S$, et al. Natural history of brain arteriovenous malformations: a long-term follow-up study of risk of hemorrhage in 238 patients. Neurosurgery 2008:63:823-9.

4. Kader A, Young WL, Pile-Spellman J, et al. The influence of hemodynamic and anatomic factors on hemorrhage from cerebral arteriovenous malformations. Neurosurgery 1994;34:801-7.

5. Khaw AV, Mohr JP, Sciacca RR, et al. Association of infratentorial brain arteriovenous malformations with hemorrhage at initial presentation. Stroke 2004;35:660-3.

6. Marks MP, Lane B, Steinberg GK, et al. Hemorrhage in intracerebral arteriovenous malformations: angiographic determinants. Radiology 1990;176:807-13.

7. Mast H, Young WL, Koennecke HC, et al. Risk of spontaneous haemorrhage after diagnosis of cerebral arteriovenous malformation. Lancet 1997:350:1065-8.

8. Redekop G, TerBrugge K, Montanera W, et al. Arterial aneurysms associated with cerebral arteriovenous malformations: classification, incidence, and risk of hemorrhage. J Neurosurg 1998;89:539-46.

9. Stapf C, Mast H, Sciacca RR, et al. Predictors of hemorrhage in patients with untreated brain arteriovenous malformation. Neurology 2006;66:1350-5.

10. Stefani MA, Porter PJ, terBrugge KG, et al. Angioarchitectural factors present in brain arteriovenous malformations associated with hemorrhagic presentation. Stroke 2002; 33:920-4.

11. Willinsky R, Lasjaunias P, Terbrugge $K$, et al. Brain arteriovenous malformations: analysis of the angio-architecture in relationship to hemorrhage (based on 152 patients explored and/or treated at the hopital de Bicetre between 1981 and 1986). J Neuroradiol 1988;15:225-37.

12. Atkinson RP, Awad IA, Batjer HH, et al; Joint Writing Group of the Technology Assessment Committee American Society of Interventional and Therapeutic Neuroradiology. Reporting terminology for brain arteriovenous malformation clinical and radiographic features for use in clinical trials. Stroke 2001;32:1430-42

13. Spetzler RF, Martin NA. A proposed grading system for arteriovenous malformations. J Neurosurg 1986;65:476-83.

14. Harrell FE Jr, Lee KL, Mark DB. Multivariable prognostic models: issues in developing models, evaluating assumptions and adequacy, and measuring and reducing errors. Stat Med 1996;15:361-87.

15. Kellner CP, McDowell MM, Haque R, et al. Analysis of hemorrhage risk factors in pediatric arteriovenous malformations. Neurosurg Focus 2011;30:A1-35.

16. Guidetti B, Delitala A. Intracranial arteriovenous malformations. Conservative and surgical treatment. J Neurosurg 1980;53:149-52.

17. Itoyama $\mathbf{Y}$, Uemura $S$, Ushio $Y$, et al. Natural course of unoperated intracranial arteriovenous malformations: study of 50 cases. J Neurosurg 1989;71:805-9.

18. Graf CJ, Perret GE, Torner JC. Bleeding from cerebral arteriovenous malformations as part of their natural history. J Neurosurg 1983:58:331-7. 
19. Crawford PM, West CR, Chadwick DW, et al. Arteriovenous malformations of the brain: natural history in unoperated patients. J Neurol Neurosurg Psychiatry 1986;49:1-10

20. Spetzler RF, Hargraves RW, McCormick PW, et al. Relationship of perfusion pressure and size to risk of hemorrhage from arteriovenous malformations. $J$ Neurosurg 1992:76:918-23.

21. Miyasaka Y, Yada K, Ohwada T, et al. An analysis of the venous drainage system as a factor in hemorrhage from arteriovenous malformations. J Neurosurg 1992; 76:239-43.

22. Langer DJ, Lasner TM, Hurst RW, et al. Hypertension, small size, and deep venous drainage are associated with risk of hemorrhagic presentation of cerebral arteriovenous malformations. Neurosurgery 1998:42:481-6.

23. Duong DH, Young WL, Vang MC, et al. Feeding artery pressure and venous drainage pattern are primary determinants of hemorrhage from cerebral arteriovenous malformations. Stroke 1998:29:1167-76.

24. Arnaout $\mathbf{0 M}$, Gross BA, Eddleman CS, et al. Posterior fossa arteriovenous malformations. Neurosurg Focus 2009;26:E12.
25. Stapf C, Mohr JP, Pile-Spellman J, et al. Concurrent arterial aneurysms in brain arteriovenous malformations with haemorrhagic presentation. J Neurol Neurosurg Psychiatry 2002;73:294-8.

26. Mansmann U, Meisel J, Brock M, et al. Factors associated with intracranial hemorrhage in cases of cerebral arteriovenous malformation. Neurosurgery 2000;46:272-9.

27. Stefani MA, Porter PJ, terBrugge KG, et al. Large and deep brain arteriovenous malformations are associated with risk of future hemorrhage. Stroke 2002:33:1220-4

28. Vinuela F, Nombela L, Roach MR, et al. Stenotic and occlusive disease of the venous drainage system of deep brain AVM's. J Neurosurg 1985;63: 180-4.

29. Maher Co, Scott RM. Linear vein-based arteriovenous malformations in children. $J$ Neurosurg Pediatr 2009:4:12-16.

30. Hofmeister C, Stapf C, Hartmann A, et al. Demographic, morphological, and clinica characteristics of 1289 patients with brain arteriovenous malformation. Stroke 2000;31:1307-10.

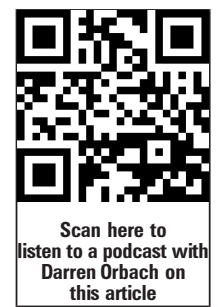

